\title{
HIGH INTENSITY PROTON BEAMS IN A MULTI-CYCLED SPS
}

\author{
A. Faugier, X. Altuna, R. Bailey, R. Blanchard, T. Bohl, H. Burkhardt, P. Collier, K. Cornelis, \\ N. Garrel, A. Hilaire, M. Jonker, R. Keizer, M. Lamont, T. Linnecar, G. de Rijk, G. Roy, \\ H. Schmickler, J. Wenninger, CERN
}

The SPS ran for 247 days during 1994; 64\% of this was with high intensity proton beam for physics data taking in the Fixed Target mode of operation, 12\% was for a lead run at the end of the year, with the remaining $24 \%$ spent in setting up and machine development. The SPS supplied LEP with 8 bunches of electrons and 8 bunches of positrons either in the 14.4 or 19.2 seconds interleaved cycling mode during the operation with protons or lead ions respectively. The new record peak intensity during the year was $3.9 \times 10^{13}$ protons per pulse at $450 \mathrm{GeV}$. A total of $11 \times 10^{18}$ proton were delivered to all targets, with an overall average during physics of $2.5 \times 10^{13}$ protons per pulse at $450 \mathrm{GeV}$. Some $6 \times 10^{18}$ protons were delivered to both neutrino experiments.

\section{SPS FIXED TARGET OPERATION}

\section{A. General}

The $450 \mathrm{GeV}$ proton operation was carried out with a cycle duration of 14.4 seconds, including four 1.2 second lepton cycles. A typical display of the different intensities delivered onto targets is shown Figure 1.

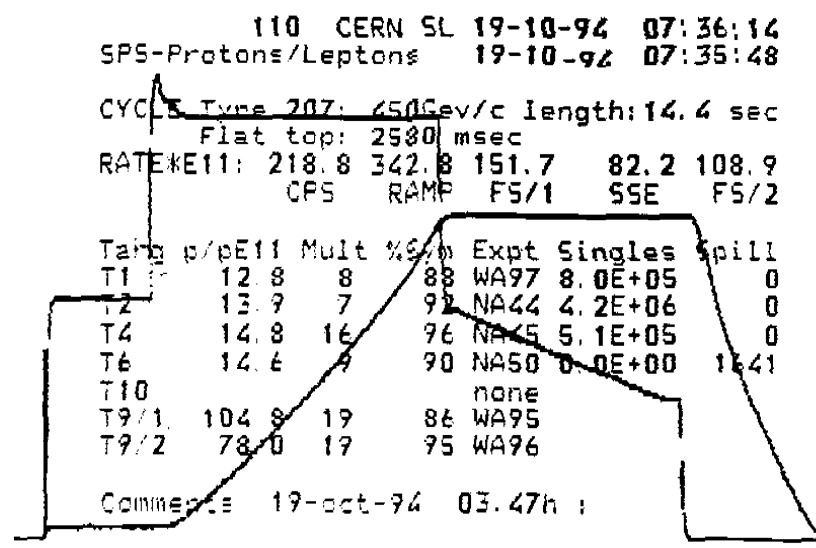

Figure 1: Proton intensities and magnetic cycle display as seen on video screens in the Control Room

The start-up period was difficult for a variety of reasons; dominant were many equipment problems, the unavailability of the injectors, and the new controls and application software following the withdrawal of the obsolete ND100 computers. The major difficulties came first from computer controls and associated software, then from the superconducting cavities, a second bi-module having been installed during the shutdown. Later, when the intensity started to increase, a high sparking rate of the West electrostatic septa (mainly tank4) caused further delays. After a careful systematic commissioning increase the intensity to $2.710^{13}$ at $450 \mathrm{GeV}$, giving $10^{13}$ ppp for the neutrino experiments. The operation was rather unstable at this moment, due partly to trips of the neutrino interlock system itself, but amplified by numerous data acquisition and controls problems (the equipment protection system philosophy was completely revised before the summer). In May/June, about 4 days of neutrino operation were lost due to numerous vacuum leaks, mainly in SPS, but also in the CPS booster. At the beginning of June, the intensity was $3.210^{13}$ at $450 \mathrm{GeV}$ of which $1.810^{3} \mathrm{ppp}$ were extracted for the neutrino target. After the June technical stop, significant improvements were observed on the computer front and the sparking in the West electrostatic septa, although not fully understood, was under control. From July, the very good transverse emittances delivered by the CPS allowed the SPS to run high intensity with a good transmission, but with a moderate overall efficiency due to a number of various faults, mainly after a second $400 \mathrm{kV}$ failure in July. The observation was made that the sparking rate on the electrostatic septa was directly affected by tiny losses during the acceleration. At the end of summer, a serious difficulty came from the superconducting cavities which suffered from frequent trips during the proton cycle, and necessitated a reduction of intensity one month before the end of the run.

A systematic study of the behaviour of the cavity and its dependence on the beam quality revealed the importance of the $14 \mathrm{GeV}$ longitudinal structure which was improved by fine adjustments of the four booster ring intensities, and of the CPS continuous transfer extraction bumpers. It was then possible to launch the common CPS-SPS intensity increase program, with the aim of finding the maximum intensity acceptable by the neutrino target (and by the machine), with minimum perturbation for the other users. The CPS was able to accelerate batches of $2.410^{3}$ protons under stable conditions and with good transverse emittances; the SPS, after some tuning to accept such a beam, was able to accelerate over $3.510^{3}$ ppp to $450 \mathrm{GeV}$, in stable conditions, reaching the peak intensity of $3.910^{13} \mathrm{ppp}$.

In conclusion, the very high intensity run at the end of the year was a success only after months of continuous effort not only in the SPS but also in the chain of injectors.

\section{B. Acceleration of high intensity proton beams}

To maintain longitudinal stability of the intense proton beam against coupled bunch instabilities the various RF cavities in the SPS must be damped, either actively or passively. Four separate RF systems, one for high intensity proton acceleration and three for lepton acceleration are present in the SPS ring. RF feedback with ona turn dalor is usad on tha fundomantol nocchond 
available for proton acceleration, while direct RF feedback is used on the three active $100 \mathrm{MHz}$ standing wave cavities used to capture long lepton bunches at injection. The twenty one $200 \mathrm{MHz}$ standing wave cavities, which accelerate lepton bunches to about $15 \mathrm{GeV}$, are passively damped in the proton cycle, the damping loop being inserted and removed once every super-cycle. The four $352 \mathrm{MHz}$ superconducting cavities, accelerating leptons to top energy, are each heavily damped using short-delay RF feedback supplemented by a one-turn delay RF feedback system [1]. All cavities have higher order mode suppressors.

The superconducting system has proved the most delicate to operate in the presence of the high intensity proton beam. This is not because of beam instabilities but because of the transient beam loading that can occur with non-uniform beams. The RF feedback essentially maintains zero voltage on the cavity, the power amplifiers injecting the current necessary to counteract the beam induced voltage. The cavity frequency, $352 \mathrm{MHz}$ is far away from the harmonics of the main accelerating frequency and for uniform beams the amplifiers are not heavily solicited. However, any strong structure on the beam has components at multiples of the revolution frequency which can increase the demand significantly. These components can excite resonances in power supplies etc., care must be taken to filter these effects, but the main problem is the extra power requirement on the amplifiers. With increasing intensity it was necessary to improve the situation in two ways; first by making the amplifiers as robust as possible, i.e. tuning the interlock circuits to the beam characteristics, and secondly by attacking the source, i.e. the beam components. The major source of the latter is the continuous transfer system in the injector which unless carefully adjusted can give beam current components to many times the revolution frequency. A secondary source is the longitudinal emittance blow-up in the injector at top energy done to prevent microwave instabilities. If this is not accurately adjusted losses can occur on parts of the beam again introducing revolution frequency components.

For the next run the current capability of the amplifier system is being increased, more improvements to the protection circuits are being added, and new digital filtering techniques are being examined which will reduce the loop response at the revolution frequency lines thus lowering the demand on the amplifiers while maintaining gain at the synchrotron frequency sidebands to prevent instability. Instrumentation to rapidly analyse the beam structure will also be introduced

\section{Neutrino beams}

During 1994, the neutrino program was again operational after a 2 year stop. The proton transfer line TT66, the neutrino target $\mathrm{T} 9$ and the neutrino beam line were completely re-build during this time. The proton beam is extracted from the machine in 2 extractions senarated hy $276 \mathrm{c}$ Roth extractions are combined $2^{\text {nd }}-4^{\text {th }}$ round off at the end of the ramp at $446 \mathrm{GeV}$. The beam is bunched with all buckets filled, except for the 220ns holes in between the injection batches. The second takes place at the end of the $450 \mathrm{GeV}$ flat top, where the beam is debunched. Both extractions have a length of $6 \mathrm{~ms}$. The beam intensity is monitored by a beam current transformer in the first part of the transfer line. The beam positions in the line are measured with secondary emision monitors, on which radiation damage was found to develop. This damage results in a non-uniform sensitivity of these detectors. To monitor the real intensity received on target an additional BCT will be added in front of the target. In the first part of 1994 the maximum allowed beam intensity on T9 for both extractions was $1.3 * 10^{13}+0.7 * 10^{13}$ protons per cycle, respectively. After a careful recalulation of the thermal and stress effects in the target these limits were lifted to $1.5^{*} 10^{13}+1.5^{*} 10^{13}$ protons per cycle. During the end of 1994 total intensities of up to $2.8 * 10^{13}$ protons per cycle on target were achieved. During a few days, $2.510^{3}$ protons were registered, and an inspection of the target, after a 3 months cooldown period revealed no visual damage.

\section{Radiation problems}

One of the very important factors linked to the high intensity running is the loss of particles in the high energy extraction channels, not only during at the time of the extraction at $450 \mathrm{Gev} / \mathrm{c}$, but also during the acceleration process.

For the survival of the equipment and to limit the personnel dose it is necessary to have the proper tools to optimize the setting-up and analyze the proper functioning of the extraction channel. Intensive studies carried out over the last 5 years have lead to the following recommendations:

1.During the annual shut-down, careful HV conditioning accompanied by minimizing the vacuum pressure by a proper bake-out to reduce the outgassing time during the start-up period;

2.During the setting-up, allow the necessary time to outgas with the beam, components like septum wires and $\mathrm{HV}$ electrodes, which receive much radiation and are likely to cause frequent sparking;

3.During operation, constant survey of the beam quality through the whole chain of accelerators involved, in order to take appropriate action at the right moment.

Application software has been developed which will allow carefully monitoring of the beam losses, normalized to the extracted intensity, to spot instantaneous changes in extraction efficiency and abnormal beamloss.

A second system of high gain beamloss detectors has been installed to monitor the evolution of the induced radioactivity and micro beamloss during acceleration.

A pilot system has allowed the development of an algorithm which calculates the present and future induced activity from the time ceries of the number of 
higher intensities will exponentially lengthen the time before access may be given to the machine in case human intervention is required Figure 2.

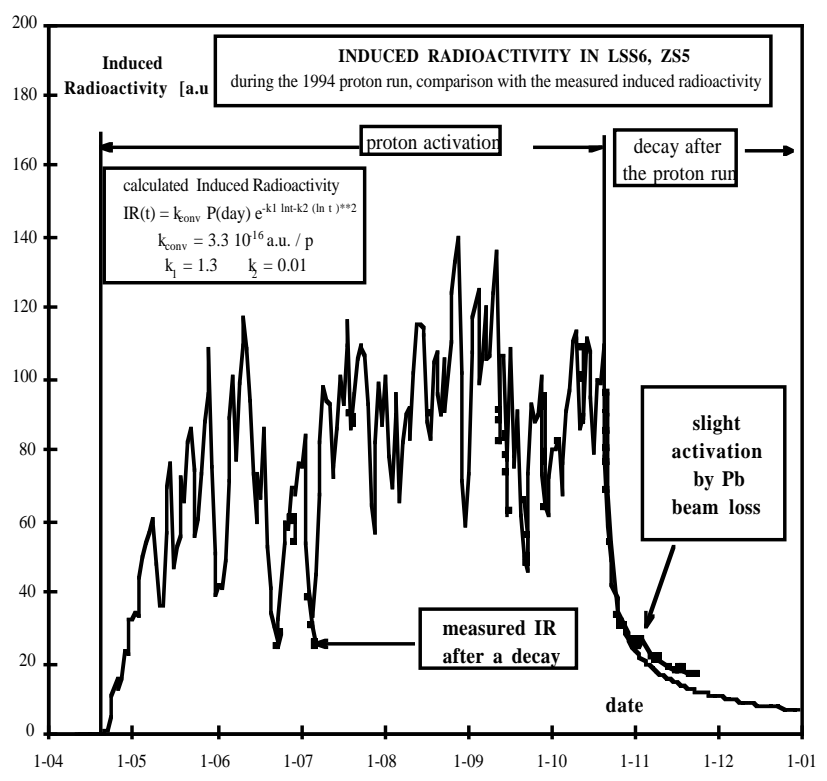

Figure 2: Measured and expected induced activity

\section{LEPTON}

The SPS and CPS nominally have $4 * 1.2$ second cycles dedicated for lepton production and transfer. In 1994 only the middle two cycles (called e+2 and e-1) were used for LEP in 8 bunch mode. The harmonic numbers of SPS and CPS were chosen such that the 8 bunches were equidistant in the SPS and no re-phasing was required by the CPS between extraction the first and second 4 bunch batches. This allowed the 8 SPS bunches to be directly injected into the 8 LEP pretzel bunches within 1 SPS cycle. In the CPS the two spare cycles were used extensively for MD, physics and the setting up and optimisation of lead ion cycles (for the SPS physics program). In the SPS less use was made of the spare time in the supercycle. One of the spare cycles (e-2) was used for commissioning of the newly installed Robinson wiggler and for optimisation of a $22 \mathrm{GeV}$ electron cycle. The other spare cycle (positron 1) saw no beam after the initial start-up period. However, the reduction in the number of active cycles in the SPS allowed the operators to concentrate on those that were left.

\section{ACKNOWLEDGEMENTS}

The work reviewed in this paper is the combined effort of a very large number of persons in the SL and PS division. It is a pleasure to thank them here. B. Desforges is in charge of the statistics; the editing and assembling has been done by M. P. Colin.

\section{REFERENCES}

[1] Improved Impedance Reduction in the CERN SPS Superconducting Cavities for High Intensity Proton Operation. D.Boussard, G.Lambert, T.P.R.Linnecar. PAC, Washington 1993 